\title{
Selecting the Appropriate Continuous Glucose Monitoring System - a Practical Approach
}

\author{
Peter Adolfsson, ${ }^{1}$ Christopher G Parkin, ${ }^{2}$ Andreas Thomas ${ }^{3}$ and Lars G Krinelke ${ }^{4}$ \\ 1. Department of Pediatrics, Kungsbacka Hospital; Institute of Clinical Sciences, The Sahlgrenska Academy, University of Gothenburg, \\ Sweden; 2. CGParkin Communications, Inc., US; 3. Medtronic Inc., Germany; 4. Dexcom, Inc., Switzerland
}

DOI: https://doi.org/10.17925/EE.2018.14.1.24

$\mathrm{T}$

wo types of continuous glucose monitoring (CGM) systems are currently available for daily diabetes self-management: real-time CGM and intermittently scanned CGM. Both approaches provide continuous measurement of glucose concentrations in the interstitial fluid; however, each has its own unique features that can impact their usefulness and acceptability within specific patient groups. This article explores the strengths and limitations of each approach and provides guidance to healthcare professionals in selecting the CGM type that is most appropriate to the individual needs of their patients.

\section{Keywords}

Real-time continuous glucose monitoring (rtCGM), intermittently scanned continuous glucose monitoring (isCGM), flash, insulin, multiple daily insulin injection (MDI), type 1 diabetes, type 2 diabetes, selfmonitoring of blood glucose (SMBG)

Disclosure: Peter Adolfsson has received a research grant from Novo Nordisk and consulting fees associated with lectures/meetings from Eli Lilly, Novo Nordisk, Abbott, Dexcom, Medtronic, Nordic Infucare, Roche and Rubin Medical. Christopher G Parkin has received consulting fees from CeQur, Dexcom, Insulet, Johnson \& Johnson, Mannkind, Roche Diabetes Care and Senseonics. Lars G Krinelke is an employee of Dexcom, Inc. Andreas Thomas is an employee of Medtronic, Inc.

Review Process: Double-blind peer review.

Compliance with Ethics: This study involves a review of the literature and did not involve any studies with human or animal subjects performed by any of the authors.

Authorship: All named authors meet the International Committee of Medical Journal Editors (ICMJE) criteria for authorship of this manuscript, take responsibility for the integrity of the work as a whole, and have given final approval to the version to be published.

open Access: This article is published under the Creative Commons Attribution Noncommercial License, which permits any non-commercial use, distribution, adaptation and reproduction provided the original author(s) and source are given appropriate credit. (C) The Authors 2018.

Received: 5 December 2017

Accepted: 21 January 2018

Citation: European Endocrinology. 2018;14(1):24-29 Corresponding Author: Christopher G Parkin, MS, CGParkin Communications, Inc., 932 Vista Lago Way, Boulder City, NV 89005, US. E: chris@cgparkin.org

Support: Funding for the development of this manuscript was provided by Dexcom, Inc., San Diego, California, US.
During the past decade, we have seen a dramatic and growing shift from traditional self-monitoring of blood glucose (SMBG) to continuous glucose monitoring (CGM). Unlike SMBG, CGM devices offer the ability to collect glucose information in a way that allows diabetes patients and their healthcare professionals to dynamically assess glucose levels and trends through a continuous stream of data. Use of CGM is universally recommended by national and international medical organisations and expert clinician consensus. ${ }^{1-4}$

Two types of CGM systems are currently available for daily diabetes self-management: real-time CGM (rtCGM) and intermittently scanned continuous glucose monitoring (isCGM), often referred to as 'flash' monitoring. Each has its own unique strengths and limitations that can impact their usefulness and acceptability within specific patient groups.

The aim of this article is help healthcare professionals understand the differences between these new technologies and provide guidance in selecting the system that is most appropriate to the individual needs of their patients.

\section{Traditional glucose monitoring \\ Glycated haemoglobin}

Glycated haemoglobin (HbA1c) remains the gold standard for assessing glycaemic control and predicting the risk of development of long-term complications by providing an average glucose level, as measured over the previous 2-3 months. It is a valuable tool for characterising population health and the primary intermediate outcome measure used by payers to assess the risk of developing complications. However, $\mathrm{HbA} 1 \mathrm{c}$ has several limitations that hinder its usefulness in daily diabetes self-management. For example, it does not reflect intra- and inter-day glycaemic excursions that may lead to hypoglycaemia or postprandial hyperglycaemia. Nor does it reflect the occurrence or degree of daily glucose variability, which has been shown to be a consistent predictor of hypoglycaemia. ${ }^{5.6}$ We also know that $\mathrm{HbA} 1 \mathrm{c}$ can be an unreliable measure during pregnancy ${ }^{\top}$ and in patients with iron deficiencies, ${ }^{8}$ anemia ${ }^{9}$ and haemoglobinopathies. ${ }^{10}$ Importantly, $\mathrm{HbA1C}$ does not provide guidance for daily adjustments in therapy.

Parameters other than $\mathrm{HbA} 1 \mathrm{c}$ may prove to be of greater importance to future risk of complications. such parameters are time in hypoglycaemia, target and hyperglycaemia ranges, frequency of hypoglycaemic events (for at least 20 minutes) and glycaemic variability (coefficient of variation $[\mathrm{CV}]$ ), as recently proposed by several international medical organisations and others.,31,12 CGM is required to perform these calculations.

\section{Self-monitoring of blood glucose}

Self-monitoring of blood glucose (SMBG) has long been considered a fundamental tool for effective management of insulin-treated diabetes, ${ }^{13-15}$ and its usefulness in non-insulin-treated diabetes is now being recognised. ${ }^{16-18}$ However, as with HbA1C measurement, SMBG also has notable limitations. First, SMBG only measures glucose at a single point in time, which provides 
no indication of the direction or velocity of changing glucose. As such, it cannot predict impending hypoglycaemia or hyperglycaemia. ${ }^{19,20}$ Moreover, SMBG devices do notautomaticallywarn patients of immediate or impending low/high glucose. Because SMBG is dependent upon the patient's decision to test, nocturnal and asymptomatic hypoglycaemia may go undetected. ${ }^{19,20}$ This is particularly concerning when the patient is sleeping or engaged in activities that require full cognition (e.g., driving a car), especially when hypoglycaemia awareness is impaired. Another limitation is the associated pain, social stigmatisation and potential for blood spill. SMBG requires a fingerstick to obtain a blood sample, which can negatively impact patient adherence to prescribed SMBG regimens.

\section{Continuous glucose monitoring}

\section{Real-time continuous glucose monitoring}

rtCGM became commercially available in 2005. Today, the Dexcom G5 ${ }^{\circledR}$ (Dexcom, Inc.) and Medtronic Enlite ${ }^{\circledR}$ (Medtronic, Inc.) systems are the two most common rtCGM systems marketed in Europe. A third system, the Abbott Navigator II (Abbott Diabetes Care) is still available; however, because it appears to be accessible in a limited number of European countries, we have not included this system in our discussion. The newest rtCGM system, Eversense (Senseonics, Inc.), features the first implantable sensor; however, we have also excluded this system from our consideration because of our limited experience with it.

\section{Description}

The Dexcom G5 and Medtronic devices consist of three components: a disposable wired sensor that is placed below the skin into the subcutaneous tissue; a transmitter that is attached to the sensor; and a receiver that displays and stores glucose information. These systems measure interstitial glucose levels and provide real-time numerical and graphical information about the current glucose level, glucose trends and trend arrows, which indicate the direction and velocity of changing glucose. Programmable alerts/alarms can be used to remind/warn patients of current and/or impending high or low glucose. Real-time alerts and alarms can be 'shared' with caregivers (currently available on the Dexcom G5 system).

The maximum indicated duration of sensor use is 6 days for Medtronic Enlite and 7 days for Dexcom G5. It is important to note that some patients have reported wearing their sensors beyond the indicated wear time, which may or may not be an issue for concern. In a recent accuracy study, Boscari and colleagues reported that the previous Dexom sensor (Dexom G4 ${ }^{\circledast}$ Platinum) performed similarly to the Abbott Libre (isCGM) sensor up to 14 days of wear ( 7 days beyond the manufacturer's indicated use). ${ }^{21}$ Nevertheless, extending sensor wear beyond its indicated time frame is considered 'off-label use'.

Both devices provide a glucose value every 5 minutes (288 values/day) and require twice-daily calibration with fingerstick blood glucose testing. Data analysis software is available for both systems: Medtronic CareLink Therapy Management Software (professional and personal versions); and Dexcom CLARITY.

It is important to note that the Dexcom G5 system has an open platform for interoperability with insulin pumps (referred to as sensor-augmented pump [SAP] therapy), smartpens and apps (e.g., Glooko, FitBit, others) and broader platforms (Diasend/Glooko). This expanded interoperability allows users to analyse their Dexcom G5 glucose data in conjunction with a variety of devices and health support apps and programmes.
The Medtronic Enlite has a closed platform and is inherently linked with the 530 insulin pump, which represents another step in SAP toward a fully closed-loop insulin infusion system. The 530G pump features software that automatically suspends insulin infusion if glucose levels drop below a certain threshold. A newer version (Medtronic 640G), features a predictive low glucose suspend function (PLGS), which suspends insulin delivery when the sensor detects impending hypoglycaemia. PLGS systems are under development by other companies.

\section{Evaluation}

Numerous recent studies have demonstrated the clinical efficacy and benefits of rtCGM use in in both children and adults with type 1 diabetes (T1D) treated with either continuous subcutaneous insulin infusion (CSII) or multiple daily insulin injection (MDI) therapy, showing improved HbA1C, shortened time spent in hypoglycaemia and hyperglycaemia and reductions in moderate to severe hypoglycaemia. ${ }^{22-29}$ It has also been shown that rtCGM during pregnancy in patients with T1D is associated with improved neonatal outcomes. ${ }^{30}$ It has therefore been suggested that rtCGM should be offered to all pregnant women with T1D using intensive insulin therapy. ${ }^{30}$

The benefits of rtCGM use have also been shown in patients with T2D treated with MDI therapy, ${ }^{31}$ in older T1D and T2D MDI-treated patients ${ }^{32}$ and in T2D who are managed with intensive insulin treatment. ${ }^{33,34}$ Importantly, in the recent I Hart CGM study, Reddy and colleagues showed that use of rtCGM more effectively reduces time spent in hypoglycaemia than isCGM among T1D patients with impaired awareness of hypoglycaemia. ${ }^{35}$

\section{Intermittently scanned continuous glucose monitoring}

At the time of writing, only one isCGM system was available, the Abbott Freestyle Libre Flash Glucose Monitoring System (Abbott Diabetes Care). The system was introduced in Europe in 2014 and a slightly different version was introduced in the US in late 2017.

\section{Description}

The Libre system continuously samples and measures interstitial glucose levels; a new glucose value is generated each minute. The sensor can provide glucose values for 14 days (10 days in US) if the patient scans at least every 8 hours. If not, the glucose information from the previous 8-hour period will be overwritten and will not available for therapy decision-making or for later download. Unlike rtCGM devices, the Libre system is factory-calibrated, which eliminates daily calibration with fingerstick testing. However, it is recommended that patients perform confirmatory blood glucose testing under the following conditions: when hypoglycaemia is detected; a trend arrow indicates rapidly changing glucose values; and symptoms are not reflected by the displayed glucose value. Patients must scan the sensor with the reader to obtain a current glucose concentration reading and to transfer stored data points from the sensor to the reader. Only glucose values obtained during the previous 8 hours are displayed in 15-minute intervals. With the current European version of the Libre system, glucose values can be scanned using the reader or an Android smartphone (NFC). Using the LiberLink app, users can share the scanned data (but not real-time) with others (e.g., spouse/partner, caregiver). In the US version, scanning is performed only with the dedicated reader. Glucose data can be downloaded for analysis, using the Abbott Freestyle Libre Software or Diasend/Glooko. The Freestyle Libre Software provides expanded options to evaluate only downloaded glucose values, whereas the Diasend/Glooko programme also synchronises information from insulin pumps and insulin pens. 
Table 1: Summary of characteristics and features of the Abbott Libre, Dexcom G5 and Medtronic Enlite systems as indicated for use outside the US

\begin{tabular}{|l|l|l|}
\hline Feature & isCGM & rtCGM \\
\hline Indicated wear life & $\begin{array}{l}14 \text { days (in EU) } \\
10 \text { days (in US) }\end{array}$ & $\begin{array}{l}6 \text { days (Medtronic) } \\
7 \text { days (Dexcom) }\end{array}$ \\
\hline Calibration & No & $2 \times /$ day \\
\hline Allows optional calibration & No & Yes \\
\hline Immediate access to glucose values & Only when & Button push \\
\hline Trend arrows & Yes & Yes \\
\hline Alerts/alarms & No & Yes \\
\hline $\begin{array}{l}\text { Insulin dosing without confirmatory } \\
\text { fingerstick testing }\end{array}$ & No (in EU) & No (Medtronic) \\
\hline Real-time remote monitoring (sharing) & No & Yes (Dexcom) \\
\hline Direct integration with insulin pump & No & Yes \\
\hline Applicable for closed-loop systems & No & Yes \\
\hline Interoperability with other devices & No & Yes \\
\hline
\end{tabular}

isCGM = intermittently scanned continuous glucose monitoring; rtCGM = real-time continuous glucose monitoring.

\section{Evaluation}

Two studies using isCGM have demonstrated significant improvements in hypoglycaemia, time in range, glycaemic variability and patient satisfaction in T1D patients ${ }^{36}$ and T2D patients treated with intensive insulin therapy; ${ }^{37}$ however, reductions in $\mathrm{HbA} 1 \mathrm{C}$ were not observed in either study. ${ }^{36,37}$ Importantly, there are some concerns regarding the accuracy in hypoglycaemic and hyperglycaemic ranges. Fokkert and colleagues recently observed lower values in the lower glycaemic ranges, and an underestimation of the effect of a meal on glucose response. ${ }^{38}$ This finding appears to be supported by the unusually high 'baseline' hypoglycaemia observed in the T2D study ${ }^{37}$ and the aforementioned Reddy study. ${ }^{35}$

\section{Patient selection}

Both rtCGM and isCGM provide patients with information about their previous and current glucose levels, as well as glucose trend and predictive information about future glycaemic status. However, there are distinct differences between these technologies, as shown in Table 1. choosing between isCGM and rtCGM should be a conscious decision, taking into account the key differences between these technologies and how they address the clinical needs, lifestyle and treatment needs of each patient.

\section{Considerations}

\section{Accuracy}

The accuracy of glucose data is a critical component of safe and effective CGM use.

A common metric for assessing CGM accuracy is the aggregate mean absolute relative difference (MARD) between all matched sensor data and reference measurements across all glucose ranges. Reported as a percentage, MARD is the average of the absolute error between all CGM values and matched reference values. However, it is important to note the limitations of using MARD values when comparing the accuracy of the various CGM systems due to differences in the methodologies and comparators used in accuracy assessments. When comparing the accuracy of two or more different CGM systems, it is advisable to perform head-to-head assessments in which the systems are running
Table 2: Percentage of matched continuous glucose monitoring reference pairs in specified glucose ranges from US Food and Drug Administration summaries of device safety and effectiveness data

\begin{tabular}{|l|l|l|l|}
\hline $\begin{array}{l}\text { CGM Glucose Range } \\
\text { mg/dL (mmol/L) }\end{array}$ & $\begin{array}{l}\text { rtCGM } \\
\text { Dexcom }\end{array}$ & \multicolumn{2}{l|}{ isCGM } \\
\hline$<40(<2.2)$ & $6.0 \%$ & $0.0 \%$ & $17 \%$ \\
\hline$\geq 40-60(\geq 2.2-3.3)$ & $74 \%$ & $37 \%$ & $23 \%$ \\
\hline$>60-80(>3.3-4.4)$ & $68 \%$ & $64 \%$ & $37 \%$ \\
\hline$>80-120(>4.4-6.7)$ & $72 \%$ & $55 \%$ & $70 \%$ \\
\hline$>120-160(>6.7-8.9)$ & $72 \%$ & $55 \%$ & $64 \%$ \\
\hline$>160-200(>8.9-11.1)$ & $59 \%$ & $50 \%$ & $58 \%$ \\
\hline$>200-250(>11.1-13.9)$ & $70 \%$ & $53 \%$ & $54 \%$ \\
\hline$>250-300(>13.9-16.7)$ & $61 \%$ & $54 \%$ & $47 \%$ \\
\hline
\end{tabular}

*Dexcom G4 system, performed with the Software 505, which is used in the Dexcom G5 Mobile system. +Calibration was performed every 12 hours. CGM = continous g/ucose monitoring; isCGM = intermittently scanned continuous glucose monitoring; rtCGM = real-time continuous g/ucose monitoring.

simultaneously in each study patient. This will neutralise the potential impact of study cohort or study setting differences. ${ }^{39}$

To our knowledge, a true 'head-to-head' comparison of the currently available rtCGM and isCGM systems has yet to be conducted. However, standardised accuracy studies published by the US Food and Drug Administration (FDA) comparing continuous glucose readings from these devices with laboratory reference values provide important insights regarding accuracy. ${ }^{40-42}$ As shown in Table 2, there are notable differences between the various CGM systems. Although the rtCGM and isCGM systems show similar concurrence within a range of $>80-200 \mathrm{mg} /$ $\mathrm{dL}(>4.4-11.1 \mathrm{mmol} / \mathrm{L})$, the accuracy of the isCGM device is concerning within the lower glucose ranges. For example, if the Libre system reads $60 \mathrm{mg} / \mathrm{dL}(2.2 \mathrm{mmol} / \mathrm{L})$, there is a $40 \%$ chance that the patient is actually between 80-160 mg/dL (4.4-8.9 mmol/L); whereas, the chance would be $6 \%$ with the Dexcom sensor. The danger here is that erroneous low sensor readings may prompt patients to treat non-existent hypoglycaemia with unnecessary carbohydrates, resulting in hyperglycaemia. This may explain why the current Libre studies showed no reductions in $\mathrm{HbA} 1 \mathrm{C}^{36,37}$

Apart from the clinical implications of CGM inaccuracy, there is the potential impact on patient persistence in using their devices. Recent evidence suggests that continued CGM use is related to patients' trust in the accuracy and reliability of the data and the usability of the device. ${ }^{43}$ Importantly, it has been suggested that patients may perceive their CGM device as more accurate and reliable when they are given support and guidance regarding how to use the data more effectively.43

\section{Alerts and alarms}

An important feature of rtCGM devices is the ability to utilise alarms and alerts that warn patients of immediate and/or impending glycaemic events. A critical concern among both clinicians and patients is severe hypoglycaemia, particularly when it occurs at night and in patients with impaired hypoglycaemia unawareness. In both the Dexcom G5 and Medtronic Enlite systems, these alarms and alerts are programmable and can be individualised to the needs of each patient. For example, if a patient has difficulty remembering to bolus before meals, an alert for rapidly rising glucose can be set at a lower level as an early warning that a bolus may have been missed. Conversely, if hypoglycaemia fear is a concern, the patient may want to set the 'rapidly falling glucose' and hypoglycaemia alerts to higher thresholds. 
An important consideration is the potential for 'alarm fatigue', which describes the condition in which patients are repeatedly disturbed by both false and frequent alarms. In many cases, patients become so frustrated that they turn their alarms off. However, as the accuracy of rtCGM has improved over the past few years, the frequency of false alerts has been significantly reduced. ${ }^{44}$ To avoid alarm fatigue, it may be advisable to have patients activate the alarm/alert functions using a step-wise approach, starting with the most important alert functions and then only the alert(s) that leads to benefit. Importantly, the alert functions should be directly related to problem solving or addressing a specific target in each individual. Through this procedure, the problem of alarm fatigue should be reduced because the patient, him/herself has chosen this to solve the problem.

\section{Data sharing}

The ability to share data with family members and caregivers is a valuable feature of both isCGM; the LibreLink app is available in Europe only, the Medtronic MiniMed Connect accessory is available in some European countries, the Dexcom G5 Mobile app is available in all countries. However, although the current isCGM device allows patients to scan their sensor with their Smartphone, sharing data is dependent upon the patient's decision to scan the sensor. As such, it does not offer the additional 'safety net' provided by the rtCGM systems, which transmit glucose values and alarms/alerts to 'followers' in real time on a continuous basis. For example, real-time sharing enables parents to remotely monitor their child's glucose levels during school, play or exercise. During night time, glucose values can be displayed in the parents' bedroom along with an added alert function. Moreover, parents or caregivers could have a separate alert setting, allowing them to proactively intervene to avoid or reduce hypoglycaemia and hyperglycaemia. This allows the parent or caregiver to act only when needed, thus avoiding the need to check glucose levels frequently or randomly during night time. The sharing function also provides important advantages for adult patients who are sleeping, driving alone, travelling or ill.

\section{Convenience}

Convenience is an important factor in sustaining CGM adherence. Thus, factory-calibration is a key advantage of the Abbott Libre system, and it is particularly desirable for patients who would like to avoid routine fingerstick blood glucose testing. However, as discussed earlier, when using Libre, patients are advised to perform fingerstick testing when hypoglycaemia is detected, when a trend arrow demonstrates a rapid change of glucose value and when symptoms are not reflected by the actual glucose value. It should also be noted that because re-calibration is not an option with the system, patients are unable to calibrate their device when glucose data do not match symptoms or confirmatory blood glucose test results. This non-ability to calibrate can affect the remaining sensor lifetime accuracy.

Ease in obtaining data is also an important convenience factor to consider. As discussed, the Abbott Libre requires patients to make a conscious decision to scan the sensor in order to obtain glucose information: glucose value, trend arrow and 8-hour trend of glucose history. For patients who desire an easy, intuitive monitoring system, this may be regarded as an advantage. However, for patients at risk for hypoglycaemia, this convenience factor is a potentially dangerous limitation, particularly among those with impaired hypoglycaemia awareness and frequent nocturnal hypoglycaemia as alerts or alarms are missing.

Another factor to consider is the sensor wear life. The 14-day sensor wear time of the Abbott Libre is a notable advantage over the rtCGM
Table 3: Recommendations for patient selection

rtCGM isCGM

- Patients treated with intensive insulin regimens (insulin pump and MDI)

- Patients with increased risk for hypoglycaemia:

- Impaired hypoglycaemia awareness

- Frequent nocturnal hypoglycaemia

- Frequent severe hypoglycaemia

- Significant glycaemic variability

- Patients who experience hypoglycaemia fear

- Patients who are physically active (especially athletes) and/or have busy lifestyles

- Patients who cannot achieve adequate glucose control with isCGM

- Patients who desire tighter glucose control

- Younger T1D patients who require continuous support by an adult (parents, school personnel and coaches in sports via 'sharing', with specific alert settings)

- Patients who want to use LGS and/or PLGS functions to reduce hypoglycaemia

- Patients with newly diagnosed T2D for episodic use as an educational tool

- T2D patients treated with noninsulintropic therapies for episodic use as an educational tool

- T2D patients who are not on intensive insulin regimens, are under good control and desire full-time or episodic isCGM as an alternative to SMBG

- Any T1D and T2D patients on insulin treatment who would like to monitor glucose without the support of alerts/alarms; and possibly transition to rtCGM in the future

- Patients who are motivated to scan their device several times per day, which is mandatory for improved glucose control (e.g., during pregnancy)

- Patients with low risk of hypoglycaemia but desire more data

- Patients who cannot afford rtCGM but desire improved glycaemic control or if a specific country specifies that isCGM is the first choice before rtCGM

isCGM = intermittently scanned continuous glucose monitoring: LGS = low glucose suspend function: $\mathrm{MDI}=$ multiple daily insulin injection: $P L G S=$ predictive low glucose suspend function; rtCGM = real-time continuous glucose monitoring; SMBG = selfmonitoring of blood glucose; T1D = type 1 diabetes; T2D = type 2 diabetes

systems, which indicate a sensor change every 6 or 7 days, depending on the device. While the extended sensor life of the Abbott Libre reduces the 'hassle' of weekly sensor replacement, the exact number of sensors lasting to 14 days is not well represented in the study literature. It should be noted that both isCGM and rtCGM can have a higher rate of inaccuracy on the first day after sensor insertion.

\section{Cost}

As with all aspects of diabetes care, cost will always be an issue. Can the patient afford the medication or the device? Is the medication or device reimbursed by the society? In the case of patient affordability, we are gratified to see that more private and public insurers are providing coverage for both isCGM and rtCGM. Nevertheless, there will always be situations where cost to the patient will be a determinant for the specific CGM device recommended. In these situations, isCGM may have an advantage over rtCGM. However, a more significant question is whether private and public insurers see an economic benefit of one technology over the other. Addressing this question will require payers to weigh the short-term against the long-term benefits. Studies that investigate the impact of sensor technologies and their long-term economic effects and health outcomes are clearly needed.

\section{Other factors}

Potential skin reactions to the sensor and/or adhesives, inadvertent sensor detachment and loss of sensor data signal are factors that should be addressed when discussing CGM use with patients. ${ }^{45}$ Problems with skin irritation and difficulties keeping the sensor/transmitter attached can limit successful CGM use. ${ }^{46}$ Although there are no definitive clinical 
studies of the occurrence of allergic reactions to the various sensors, a review of the FDA Manufacturer and User Facility Device Experience (MAUDE) database raises some concerns about the Libre system. ${ }^{47}$ Analysis of the MAUDE database for the month of December showed that a significantly higher percentage of Libre users reported an allergic reaction to their sensor compared with Dexcom users: $0.30 \%$ versus $0.01 \%$, respectively. Therefore, patients (especially those with sensitive skin) who are considering glucose monitoring with the Libre system should be counselled about this potential problem. The duration of the time period when a sensor is to be used can also affect the incidence of skin problems. Longer durations of use will require more adhesives in spite of external influences (e.g., pressure, impact, sweat, bathing).

Regarding adhesives, all patients should be educated on skin care and prepare the skin before the use of any sensor. Finding the right combination of supplemental products (liquid adhesive, adhesive wipes, tape, wraps) can improve successful CGM wear and acceptance, especially in young patients with diabetes. ${ }^{46}$

Another factor to consider and discuss with patients is potential sensor signal disruption. ${ }^{39}$ It is important to explain to patients that signal disruption is often the result of acute compression forces (e.g., during sleep) on the sensor-surrounding tissue, impacting micro-circulation, and not an indication of sensor inaccuracy.

\section{Recommendations}

To date, no definitive studies have been conducted to determine the most appropriate patient characteristics for rtCGM versus isCGM. However, based on our review of these technologies, we have compiled a list of general guidelines for matching patients with the CGM system that may best meet their needs. (Table 3) It is important to note that our recommendations are intended only as a starting point. As with all aspects of diabetes management, treatment must be individualised to the clinical situation, lifestyle, motivation, cognitive capabilities and socioeconomic status of each patient. Moreover, the choice between the different CGM systems is sometimes about reimbursement and financial (bidding) decisions based on society's healthcare system, when clinically proven benefits contrast with limited investment in healthcare. Healtheconomic studies are needed for informed decision-making.

\section{Summary}

Managing diabetes is a complex and often frustrating challenge. For patients, success in their daily self-management can be unpredictable and discouraging, despite their best efforts. For healthcare professionals, lack of reliable glucose information restricts their ability to monitor their patients' glycaemic status and make informed decisions about therapy adjustments. Evolving tools and technologies such as CGM may relieve some of this burden for both patients and their healthcare professionals.

In our view, both isCGM and rtCGM offer clear advantages over SMBG by providing considerably more robust and useable information. Some patients will do well with isCGM long-term, and it may provide an option as an initial step toward rtCGM. This may enable some patients to become more comfortable using glucose data, particularly glucose trends and rate of change arrows for everyday decision-making, which could lead to better outcomes. However, the limitations of isCGM (e.g., lack of alarms/alerts, intentional scanning, inability to re-calibrate) should always be a consideration.

Conversely, rtCGM represents more advanced technology for glycaemic management, providing the ability to view current glucose levels, glucose trends and direction/rate of glucose automatically in real time. Importantly, the alarms and alerts featured in rtCGM systems allow patients to detect and immediately act upon immediate and impending glycaemic events. Additionally, rtCGM is the only alternative for patients who wish to utilise SAP, low glucose suspend (LGS) and PLGS therapy, and it is currently the only alternative for fully closed-loop insulin delivery systems.

As with all new technology, safe and effective use of isCGM and rtCGM devices requires thorough education/training in their utilisation and technological limitations. ${ }^{48}$ To support this need, Abbott, Dexcom and Medtronic offer comprehensive instructional programmes on their websites and in printed formats. We strongly recommend that clinicians and patients utilise these materials. We also urge the diabetes research community to focus efforts on generating data that will facilitate matching the specific CGM technologies with appropriate patient populations, and to evaluate the short- and long-term economic impact of these technologies to provide guidance to both healthcare professionals and payers.
1. American Diabetes Association, 6. Glycemic Targets: Standards of Medical Care in Diabetes-2018. Diabetes Care. 2018;41(Suppl 1):S55-S64.

2. Borot S, Benhamou PY, Atlan C, et al. Practical implementation, education and interpretation guidelines for continuous glucose monitoring: A French position statement. Diabetes Metab. 2017; pii:S1262-3636(17)30550-5. [Epub ahead of print].

3. Danne T, Nimri R, Battelino T, et al. International Consensus on Use of Continuous Glucose Monitoring. Diabetes Care. 2017:40:1631-40.

4. Fonseca VA, Grunberger $G$, Anhalt $H$, et al. for the Consensus Conference Writing Committee. Continuous Glucose Monitoring: A Consensus Conference of the American Association of Clinical Endocrinologists and American College of Endocrinology. Endocr Pract. 2016;22:1008-21.

5. Cox DJ, Kovatchev BP, Julian DM, et al., Frequency of severe hypoglycemia in insulin dependent diabetes mellitus can be predicted from self-monitoring blood glucose data. J Clin Endocrinol Metab. 1994;79:1659-62.

6. Qu Y, Jacober SJ, Zhang Q, et al. Rate of hypoglycemia in insulin-treated patients with type 2 diabetes can be predicted from glycemic variability data. Diabetes Technol Ther. 2012;14:1008-12.

7. Nielsen $L R$, Ekbom P, Damm P, et al. HbA1C levels are significantly lower in early and late pregnancy. Diabetes Care. 2005;27:1200-1.

8. Ford ES, Cowie CC, Li C, et al. Iron-deficiency anemia non-iron-deficiency anemia and HbA1C among adults in the US J Diabetes. 2011;3:67-73.

9. National Diabetes Information Clearinghouse (NDIC), Sickle cell trait and other hemoglobinopathies and diabetes: important information for providers. Available at: http://diabetes.niddk nih.gov/dm/pubs/hemovari-A1C/index.aspx (accessed 26 August 2017).
10. Bry L, Chen PC, Sacks DB. Effects of hemoglobin variants and chemically modified derivatives on assays for glycohemoglobin Clinical Chemistry. 2001:47:153-63.

11. Agiostratidou $G$, Anhalt $H$, Ball $D$, et al. Standardizing Clinically Meaningful Outcome Measures Beyond HbA1c for Type 1 Diabetes: A Consensus Report of the American Association of Diabetes. A Consensus Report of the Amercan Association of Clinical Endocrinologists, the American Association of Diabetes Society, JDRF International, The Leona M. and Harry B. Helmsley Society, JDRF International, The Leona M. and Harry B. Helmsley Charitable Trust, the Pediatric Endocrine So
Exchange. Diabetes Care. 2017;40:1622-30.

12. Wright LA, Hirsch IB. Metrics beyond hemoglobin A1C in diabetes management: time in range, hypoglycemia, and other parameters. Diabetes Technol Ther. 2017;19 (Suppl 2):S16-S26.

13. Diabetes Control and Complications Trial Research Group. The effect of intensive treatment of diabetes on the development and progression of long-term complications of insulindependent diabetes mellitus. N Eng/ J Med. 1993;329:977-86

14. Miller KM, Beck RW, Bergenstal RM, et al. T1D Exchange Clinic Network. Evidence of a strong association between frequency of self-monitoring of blood glucose and hemoglobin A1c levels in T1D exchange clinic registry participants. Diabetes Care. in T1D exchange

15. Kato N, Cui J, Kato M. Structured self-monitoring of blood glucose reduces glycated hemoglobin in insulin-treated diabetes. J Diabetes Investig. 2013;4:450-3

16. Polonsky WH, Fisher L, Schikman CH, et al. Structured selfmonitoring of blood glucose significantly reduces A1C levels in poorly controlled, noninsulin-treated type 2 diabetes: results from the Structured Testing Program study. Diabetes Care. 2011;34:262-7.

17. Franciosi M, Lucisano G, Pellegrini F, et al. ROSES: role of self-monitoring of blood glucose and intensive education in patients with type 2 diabetes not receiving insulin. A pilot randomized clinical trial. Diabet Med. 2011:28:789-96.

18. Kempf K, Kruse J, Martin S. ROSSO-in-Praxi follow-up: longterm effects of self-monitoring of blood glucose on weight, hemoglobin $A 1 C$ and quality of life in patients with type 2 hemoglobin A1c, and quality of life in patients with type 2

19. Bolli B Hypoglycaemia unawareness Diabetes Metab. 1997:23:29-35.

20. Gold AE, Macleod KM, Frier BM. Frequency of severe hypoglycemia in patients with type I diabetes with impaired awareness of hypoglycemia. Diabetes Care. 1994;17:697-703.

21. Boscari F, Galasso S, Facchinetti A, et al. Freestyle Libre and Dexcom G4 Platinum sensors: accuracy comparisons during two weeks of home use and use during experimentally induced glucose excursions. Nutr Metab Cardiovasc Dis. 2017; pii: S0939-4753(17)30263-6. [Epub ahead of print].

22. Lind M, Polonsky W, Hirsch IB, et al. Continuous glucose monitoring vs conventional therapy for glycemic contro in adults with type 1 diabetes treated with multiple daily insulin injections: the GOLD randomized clinical trial. JAMA . 2017;317:379-87.

23. Aleppo G, Ruedy KJ, Riddlesworth TD, et al. REPLACE-BG: A randomized trial comparing continuous glucose monitoring with and without routine blood glucose monitoring in adults with well-controlled type 1 diabetes. Diabetes Care. 2017;40:538-45.

24. Beck RW, Riddlesworth T, Ruedy K, et al. for the DIAMOND Study Group. Effect of continuous glucose monitoring on glycemic control in adults with type 1 diabetes using insulin injections: The DIAMOND Randomized Clinical Trial. JAMA. 2017;317:371-8.

25. Riddlesworth T, Price D, Cohen N, Beck RW. Hypoglycemic event frequency and the effect of continuous glucose monitoring. in adults with type 1 diabetes using multiple daily insulin injections. Diabetes Ther. 2017;8:947-51. 
26. Polonsky WH, Hessler D, Ruedy KJ, Beck RW, for the DIAMOND Study Group. The impact of continuous glucose monitoring on
markers of quality of life in adults with type 1 diabetes: further markers of quality of life in adults with type 1 diabetes: further Care. 2017:40:736-41

27. Šoupal J, Petruželková L, Flekač M, et al. Comparison of different treatment modalities for type 1 diabetes, including sensor-augmented insulin regimens, in 52 weeks of follow-up: a COMISAIR study. Diabetes Technol Ther. 2016;18:532-8.

28. Šoupal J, Petruželková L, Flekač M, et al. The combination of rt-CGM and MDI can be a suitable alternative to Sensor Augmented Pump therapy for some patients. Presented at: European Association for the Study of Diabetes (EASD) 2017 Annual Meeting, Lisbon, Portugal, 11 September 2017.

29. van Beers CA, DeVries JH, Kleijer SJ, et al. Continuous glucose monitoring for patients with type 1 diabetes and impaired awareness of hypoglycaemia (IN CONTROL): a randomised, openlabel, crossover trial. Lancet Diabetes Endocrinol. 2016;4:893-902.

30. Feig DS, Donovan LE, Corcoy R, et al. Continuous glucose monitoring in pregnant women with type 1 diabetes (CONCEPTT): a multicentre international randomised controlled trial. Lancet 2017:390:2347-59.

31. Beck RW, Riddlesworth TD, Ruedy K, et al., for the DIAMOND Study Group. Continuous glucose monitoring versus usua care in patients with type 2 diabetes receiving multiple daily insulin injections: a randomized trial. Ann Intern Med. 2017;167:365-74

32. Ruedy KJ, Parkin CG, Riddlesworth TD, Graham C, for the DIAMOND Study Group. Continuous glucose monitoring in older adults with type 1 and type 2 diabetes using multiple daily injections of insulin: Results from the DIAMOND trial,
J Diabetes Sci Technol. 2017:11:1138-46.

33. YoO HLAn HG Park SY, et al. Use of a real time continuous glucose monitoring system as a motivational device for poorly controlled type 2 diabetes. Diabetes Res Clin Pract. 2008;82:73-9.

34. Vigersky RA, Fonda SJ, Chellappa M, et al. Short- and long-term effects of real-time continuous glucose monitoring in patients with type 2 diabetes. Diabetes Care. 2012;35:32-8.

35. Reddy M, Jugnee N, El Laboudi A, et al. A randomised controlled pilot study of continuous glucose monitoring and Flash glucose monitoring in people with type 1 diabetes and impaired awareness of hypoglycaemia. Diabet Med. 2017; doi: 10.1111/dme.13561. [Epub ahead of print].

36. Bolinder J, Antuna R, Geelhoed-Duijvestijn P, et al. Novel glucose-sensing technology and hypoglycemia in type 1 diabetes: a multicentre, non-masked, randomised controlled trial. Lancet. 2016;388:2254-63.

37. Haak $T$, Hanaire $H$, Aijan R, et al. Flash glucose-sensing technology as a replacement for blood glucose monitoring for the management of insulin-treated type 2 diabetes: a multicenter, open-label randomized controlled trial. Diabetes Ther. 2017;8;55-73.

38. Fokkert MJ, van Dijk PR, Edens MA, et al. Performance of the Freestyle Libre Flash glucose monitoring system in patients with type 1 and 2 diabetes mellitus. BMJ Open Diabetes Res Care. 2017;5:e000320. doi:10.1136/bmjdrc-2016-000320.

39. Schoemaker M, Parkin CG. CGM-How Good Is Good Enough? In: Kirchsteiger H, Jørgensen J, Renard E, del Re L (eds). Prediction Methods for Blood Glucose Concentration. Lecture Notes in Bioengineering. Cham: Springer, 2016.

40. US Food and Drug Administration. Summary of safety and effectiveness data (SSED). Dexcom G4 PLATINUM Continuous Glucose Monitoring system. Available at: www.accessdata. fda.gov/cdrh_docs/pdf12/P120005S018b.pdf (accessed 3 December 2017).

41. US Food and Drug Administration, Summary of safety and effectiveness data (SSED), Medtronic MiniMed 530G System (Enlite Sensor). Available at: www.accessdata.fda.gov/cdrh docs/pdf12/p120010b.pdf (accessed 19 January 2018).

42. US Food and Drug Administration, Summary of safety and effectiveness data (SSED). Freestyle Libre Flash Glucose Monitoring System. Available at: www.accessdata.fda.gov/ cdrh_docs/pdf16/P160030B.pdf (accessed 3 December 2017).

43. Polonsky WH, Hessler D. Perceived accuracy in continuous glucose monitoring: understanding the

44. Peyser TA, Nakamura K, Price D, et al. Hypoglycemic accuracy and improved lu and improved low glucose alerts of the latest Dexcom G4 Technol Ther. 2015;17:548-54.

45. Petrie JR, Peters AL, Bergenstal RM, et al. Improving the clinical value and utility of CGM systems: issues and recommendations. Diabetes Care. 2017, 10.1614-21.

46. Englert K, Ruedy K, Coffey J, et al. Skin and adhesive issues with continuous glucose monitors: a sticky situation. J Diabetes Sci Technol. 2014;8:745-51.

47. US Food and Drug Administration, MAUDE - Manufacturer and User Facility Device Experience. Available at: www.accessdata. fda.gov/scripts/cdrh/cfdocs/cfmaude/search.cfm (accessed 17 January 2018).

48. Rodbard $D$. The challenges of measuring glycemic variability. J Diabetes Sci Technol. 2012;6:712-5. 